\title{
Social dynamics of energy behaviour
}

\author{
Energy is consumed in social environments and in the presence of social peers. But social interactions do not just \\ happen alongside energy behaviour - the two are intrinsically linked.
}

P eople do not only consume energy in isolation. Many if not most energy consumption activities are social activities. For instance, decisions about how to heat homes, workplace and public spaces affect the thermal comfort of many people who may have different comfort needs or financial constraints tied to utility bills. However, programmes and policies aimed at reducing energy consumption treat the home, office or building as the unit of analysis, with little consideration of the people within these structures and how they interact with each other.

Yet, navigating these different preferences is an inherently social problem. Even seemingly mundane daily interactions such as a discussion between spouses about whether the house feels hot or cold has energy implications if it results in a thermostat adjustment ${ }^{1}$. Interventions to reduce home energy consumption may be ineffective if they do not consider the social dynamics involved in communicating about and coordinating home heating or cooling behaviour amongst housemates ${ }^{2}$.

This Focus issue brings together pieces that explore the complexity of social relations and interactions that impact energy behaviour. As Tom Hargreaves and Lucie Middlemiss illustrate in their Perspective, these interactions extend beyond explicit energy-relevant behaviours such as managing home temperature or adopting energy efficient technology. Rather, social relations fundamentally impact all aspects of daily routines and consequently when, why and how people consume energy. For instance, energy demand in families of school-age children is constrained by the fixed time of the school day, homework and after-school activities.

Family relations within a household is just one type of social relation that shapes energy behaviour. Relations between landlords and tenants can impact energy efficiency if tenants are reluctant to raise weatherization concerns with their landlord for fear of being evicted; a lack of trust in relations between the public and energy suppliers can lead to low uptake of smart meters; low-income households may be reluctant to lead a community energy initiative if such projects are perceived as 'belonging' to the middle class. Hargreaves and Middlemiss draw on these and other rich examples to develop a typology of social relations that influence energy demand: those with family and friends, with agencies and communities, and those associated with social identities. This offers a starting point for better understanding energy demand needs and how they change over time, which can lead to more effective policy and intervention design.

Social identity can be defined in a myriad of ways, such as in terms of the geography of where one lives, the social networks within which one operates, demographic characteristics or the values one holds. These social identities in turn denote group membership, which can also have implications for energy behaviour in the form of peer-effects: people often adopt energy saving behaviours because others in their peer-group are doing so, either because of direct interpersonal communication or because of shifting social norms. For instance, households are more likely to adopt rooftop solar power if their neighbours have ${ }^{3}$, and comparing a household's electricity bill to that of its neighbours has been shown to reduce home energy use ${ }^{4}$.

In a Review, Kimberly Wolske, Kenneth Gillingham and P. Wesley Schultz synthesize the literature on peer effects, and discuss the different pathways through which social influence can give rise to them. In doing so they highlight the value of integrating thinking across economics - which has provided clear causal evidence of peer effects in the energy domain - with psychology, sociology and communication studies - which provide insights into how and why these effects might occur - for generating predictions about which social processes can be leveraged to promote different energy behaviours.

Finally, in a Comment, Larissa Nicholls, Yolande Strengers and Jathan Sadowski flip the discussion: instead of considering how social dynamics affect energy use, the researchers discuss the potential for emerging smart home technologies that promise energy savings to influence social interactions in negative ways.

Some of these impacts are obviously nefarious. For instance, smart technologies offer control to the household member who is more technologically savvy at the expense of another, which can exacerbate existing power imbalances. But other negative impacts may be less apparent. For example, although parents may be relieved to avoid fighting with teenagers over turning off appliances that are not in use, the absence of these interactions removes the opportunity for social transmission of energy efficiency values and practices.

The pieces in this Focus draw from a range of social science disciplines and approaches to illustrate how social interactions impact and are impacted by energy, from quantitative studies in psychology and economics to qualitative work in sociology and human geography. We hope that publishing these pieces together will encourage readers to look beyond their traditional disciplinary and methodological boundaries, and draw connections that inspire new lines of academic inquiry.

At the same time, although they approach the social dynamics of energy from different perspectives, all of the pieces in this Focus highlight that attempts to promote sustainable energy transitions through policies and interventions will only be effective if they are rooted in a deeper understanding of the complex social relations and interactions underlying energy behaviour.

Published online: 18 March 2020 https://doi.org/10.1038/s41560-020-0595-8

\footnotetext{
References

1. Sintov, N. D., White, L. V. \& Wapole, H. PLoS ONE 14 e0224198 (2019).

2. Outcault, S., Sanguinetti, A. \& Pritoni, M. Energy Res. Soc. Sci. 45, 276-286 (2018)

3. Bollinger, B. \& Gillingham, K. Market. Sci. 31, 900-912 (2012).

4. Allcott, H. J. Public Econ. 95, 1082-1095 (2011).
} 